\title{
A Multi-Objective Model for a Multi-Period Distribution Management Problem
}

\author{
Rita Ribeiro* \\ Universitat Pompeu Fabra
}

\author{
Helena R. Lourenço* \\ Universitat Pompeu Fabra
}

February 2001

\begin{abstract}
The problems arising in commercial distribution are complex and involve several players and decision levels. One important decision is related with the design of the routes to distribute the products, in an efficient and inexpensive way.

This article deals with a complex vehicle routing problem that can be seen as a new extension of the basic vehicle routing problem. The proposed model is a multi-objective combinatorial optimization problem that considers three objectives and multiple periods, which models in a closer way the real distribution problems. The first objective is cost minimization, the second is balancing work levels and the third is a marketing objective. An application of the model on a small example, with 5 clients and 3 days, is presented. The results of the model show the complexity of solving multi-objective combinatorial optimization problems and the contradiction between the several distribution management objectives.
\end{abstract}

\footnotetext{
*Adress: Departamento d'Economia i Empresa, Universitat Pompeu Fabra, Ramon Trias Fargas 25-27, 08005 Barcelona, Spain. rita.ribeiro@econ.upf.es helena.ramalhinho@econ.upf.es
} 


\section{Introduction}

The growing number of problems that firms are facing nowadays with relation to the distribution of their products and services, has lead logistics to be of primary concern to many industries.

The importance of good distribution strategy in today's competitive markets can not be overstressed. In many industries an important component of the distribution systems is the design of the routes of vehicles to serve their client's demand.

New trends in the supply-chain management are, as pointed out by some industry leaders, "..better customer service...greater customer sophistication" (OR/MS Today, August 2000). Customer service is becoming more important, clients demand more than a product, they demand a product arriving on time, an easy ordering system or a just-in-time distribution.

In this work, we will explore the decision making problems in distribution management related with the delivery of products to customers, on a given period of time, using a fleet of vehicles. The decisions on how to assign customers to drivers and to design the routes made by each vehicle constitute the Vehicle Routing Problem (VRP). The motivation to work on this VRP arises by the distribution problems faced by the food and beverage industry.

Vehicle routing problems have been explored both in the management and operations research literature. The models found in the latter literature are often away from reality, since they do not consider issues present in real distribution, as for example multiperiod planning. To get closer to real world problems and to reflect the multitude of concerns in distribution management we will extend the basic VRP. The final result of this extension will be a multiobjective model that takes into consideration three different objectives. These objectives are:

1- Cost objective

2- Human resource management objective,

3- Marketing objective

The idea is to do a cross-function planning in the supply-chain management by including in the model decisions that belong to different areas of a firm.

The first objective is the classical objective of VRP, which consists of minimizing total cost. However, this objective is often object of criticism by the users and planners, since it does not take into consideration other strategies of the company as, for example, customer service. The second objective expresses the need for balancing work levels. This objective is related with the human resources management which is a growing source of competitiveness in today's firms. The third expansion of the model is the one that tries to implement a marketing policy. In a growing competitive environment many firms adopt strategies of tight relationships with their customers where loyalty and friendship play a key role. As stated by Lee \& Ueng (99), "in planning vehicle routing transportation, companies are pursuing an all win strategy (organizationemployees-customers) adding the employee work load-balancing factor".

The present article is organized in the following way: In the next section we 
will present a review of the VRPs and a formulation of the basic model. Section 3 will discuss the extensions of the basic model and an approach for the multiobjective VRP. In section 4, we will show the implementation of the model on a small example and the results obtained are analyzed. The difficulty to solve these problems on a real world case leads us to the need for an heuristic approach. This approach will be the subject of our future research and is explained in section 6. Finally, in the fifth chapter the conclusions of the work are presented.

\section{Vehicle routing problems}

Vehicle routing decisions are extremely important within a company to maintain its competitiveness an allow it to best exploit the available resources and to distribute its products at the lowest possible cost.

Significant amount of research efforts have been dedicated to VRP, see for example Crainic \& Laporte (98). The most well known is a basic VRP which can be briefly defined as the following: given a set of customers with known demand and location, define a set of routes, starting and finishing at one depot, that visits all customers with minimal cost. A more detailed formulation will be presented next, since the basic VRP model is the starting point for any study in VRP.

Despite the research efforts in academic research on VRP, there still exists a considerable gap in the application in practical problems of the models and techniques developed on the academic side.

After, we will briefly review different models and solution techniques for the VRP.

\subsection{The basic model for the VRP.}

The basic model of the vehicle routing problem considers a set of nodes, representing retailers or customers, at a known location, that must be served by one depot. Each node has a known demand. A set of vehicles $K$, with equal capacity is available to serve the customers. The routes must start and finish at the depot. The objective is to define the set of routes to serve all customers with minimal cost.

For each pair of nodes, a fixed known cost is associated. We assume this cost matrix is symmetric and can represent a real cost, distance or time. The main constraints of the problem are that all the demand must be satisfied and the vehicles capacity can not be exceeded.

There are several formulations of the classical VRP in the literature, for some of these formulations see Fisher \& Jaikumar (78) (81), Kulkarni \& Bhave (85) and Gouveia (95). The integer linear programming formulation described next was proposed by Fisher \& Jaikumar (78) and Fisher \& Jaikumar (81). Consider the following data:

$I=1, \ldots, n \quad$ set of nodes, that correspond to the different locations of the customers, node 1 corresponds to the depot.

$K=1, \ldots, m \quad$ set of vehicles; 
$Q \quad$ capacity of each vehicle;

$q_{i} \quad$ demand of customer $\mathrm{i}, i=1, \ldots, n$;

$c_{i j} \quad$ cost of going from $\mathrm{i}$ to $\mathrm{j}, i=1, \ldots, n ; j=1, \ldots, n$.

This formulation considers two types of variables:

$x_{i j k}= \begin{cases}1, & \text { if vehicle } k \text { visits customer } j \text { immediatly after customer } i \\ 0, & \text { otherwise }\end{cases}$

$y_{i k}= \begin{cases}1, & \text { if customer } i \text { is visited by vehicle } k \\ 0, & \text { otherwise }\end{cases}$

The formulation of the problem is as follows:

Objective Function :

$$
\operatorname{Min} \sum_{i=1}^{n} \sum_{j=1}^{n} c_{i j} \sum_{k=1}^{m} x_{i j k}
$$

Subject to :

$$
\begin{aligned}
& \sum_{k=1}^{m} y_{i k}=1, \quad \forall i=2, \ldots, n \\
& \sum_{k=1}^{m} y_{1 k}=m \\
& \sum_{i=2}^{n} q_{i} y_{i k} \leq Q, \quad \forall k=1, \ldots, m \\
& \sum_{j=1}^{n} x_{i j k}=\sum_{j=1}^{n} x_{j i k}=y_{i k}, \\
& \forall i=2, \ldots, n ; k=1, \ldots, m \\
& \sum_{j, i \in S} x_{i j k} \leq|S|-1 \text {, } \\
& \forall S \text { non-empty subset of }\{2, \ldots, n\} ; k=1, \ldots, m \\
& x_{i j k} \in\{0,1\} ; y_{i k} \in\{0,1\} \text {, } \\
& \forall i=1, \ldots, n ; k=1, \ldots, m
\end{aligned}
$$

Constraint (1) ensures that each customer is visited by one vehicle only. Constraint (2) guarantees that all vehicles visit the depot. Constraint (3) represents the vehicle capacity constraint. For each vehicle $k$, we guarantee that the sum of the demand of the nodes that the vehicle covers is less or equal to its maximum capacity. Here we assume that none of the clients has a daily demand that exceeds Q. The fourth constraint ensures that if a vehicle visits a client it also has to leave the client.

Constraint (5) is the sub-tour elimination constraint. This constraint implies that the arcs selected contain no sub-cycles. It states that for every vehicle, the following holds: for every non-empty subset $S$ of $\{2, . ., n\}$, the number of arcs that are in the route of this vehicle, with both nodes belonging to $S$, has to be less or equal to the number of elements of $S$ minus 1 . 
The last constraint defines the variables $x$ and $y$ as binary. The objective function is minimizing the total distance(or cost) of the routes.

The basic VRP is a generalization of the Traveling Salesman Problem, where more than one vehicle is available, for TSP references see for example Lawer et al.(85).

The TSP belongs to the class of NP-hard (non-deterministic polynomial time) problems, and so does the basic VRP and extensions. This means that the computational complexity of the problem grows exponentially with its size, i.e., it grows exponentially with the number of clients and vehicles.

Therefore, most of the work on the basic and extensions are on developing optimization models and algorithms and heuristic approaches to solve them. There are several works where extensions of basic VRP, new formulations and models are presented within the context of vehicle routing. And, other works where exact methods or heuristic approaches are described. Due the complexity of the VRP usually exact methods are only able to solve small instance, meanwhile, for larger instances heuristic techniques are applied. Next we will present a brief review of VRP related research.

The review will not be exhaustive, for a more complete analysis see the survey articles on VRP by Laporte and Osman (95), Laporte (92), Desroisers et al (96) and Fisher (96) and the book of Crainic and Laporte(98). An extensive list of VRP research papers can be found on http://www.imm.dtu.dk/ 〜orgroup/VRP_ref/.

\subsection{Extensions of the basic VRP}

Next we review some important work on the area of vehicle routing. The VRP was first proposed by Dantzing and Ramser (59).

One way to classify the extensions of the VRP could be to divide them into the following types:

- Single depot

- Multiple depot

- Time windows

- Multiple use of vehicles

- Backhauls

- Heterogeneous fleet

- Satellite facilities

- Open VRP

- Stochastic VRPs

The single depot model represents the basic formulation of the VRP presented before. If a set of depots is considered we are in the presence of a 
Multi-depot Vehicle Routing problem (MDVRP), see for example, Dell'Amico, Fischetti \& Toth (93). Another generalization of the VRP is the introduction of time windows constraints. In the VRP with time windows we have to ensure that the service at any customer starts within a given time interval, called a time window, (see Desrochers, Desrosier and Solomon (92)) Kohl, Desrosiers, Madsen, Solomon and Soumis (99) proposed an optimization algorithm to this problem. Another version of this extension is the VRP with soft time windows, where vehicles can arrive before a lower bound or after an upper bound. Taillard et al.(97) propose a heuristic for this specific problem.

The basic version assumes that a vehicle can be loaded only once during a period of time. The elimination of this assumption leads us to what is known as the VRP with multiple use of vehicles. In this model, the vehicle can be used more than once in a time period. Taillard, Laporte and Gendreau (96) and Brandão and Mercer (97) proposed several heuristics to solve this problem.

The VRP with Backhauls consists of a VRP where the customers are divided into two subsets: the linehauls and the backhauls. Backhauls customers have to be visited after linehaul customers. Mingozzi et al (99) review the literature on these problems.

Another type of problems is the VRP with heterogeneous fleet, where, $n_{k}$ vehicles of type $k$ are available, $d_{i j k}$ represents the cost of traveling from one node to another with vehicle $k$, and the use of a vehicle of type $k$ implies a fixed cost $f_{k}$. Different vehicle types will reflect different fixed costs. The goal is to determine a fleet of vehicles such that the sum of fixed and travel costs is minimized. Ochi et al (98) and Taillard (96) have developed algorithms to solve this problem.

In some cases firms may have apart from the depot other facilities that can be used to fill the vehicles. This satellite replenishment allows drivers to continue making deliveries without returning to the depot. This problem is known as VRP with satellite facilities and can be found in Bard, Huang and Jaillet (98).

Many firms decide to subcontract part or all of their transportation services. In these cases the constraint that forces the vehicles to go back to the depot may make no sense. The formulation of this problem has been presented by Sariklis and Powell (00) and is known as the Open Vehicle Routing Problem (OVRP).

All the above versions are based on the assumption that all the parameters of the model are known in advance. But, in many cases, there can be elements that have some degree of uncertainty. These cases are referred to in the literature as Stochastic Vehicle Routing Problems (SVRP). In some cases firms can only know the demand when they have arrived to a client location. So, the problem is in not satisfying a client or in coming back to the depot to avoid going out of stock. There are also problems where customer locations are stochastic. Others consider uncertainty in travel times. This applies, for example, in distributing inside a city, depending on the hours we are traveling, travel time may vary. See Gendreau, Laporte and Séguin (96) for a summary of the scientific literature on SVRP.

Another important reference is the work by Lee and Ueng (99), where they 
extend the basic VRP to a multi-objective model, with two objectives: Minimizing cost and balance workload.

All the above extensions try to approximate models to reality. Each firm faces specific distribution characteristics making it impossible to explore all the variations of a routing problem. Nevertheless, we can find some works on real applications of the VRP. The work of Golden and Wasil (87) about vehicle routing on the soft drink industry and the work of Atkinson (98) on VRP with time windows applied to the distribution of school meals within a large urban area, among others.

In this article we will try to get closer to the management concerns and extend the VRP to solve a specific distribution problem, considering routing decisions for several periods on a three objectives approach.

As mentioned, the motivation was distribution problems arising in food and beverage industry. In this industries, the distribution plan is usually done weekly. Also, the relationship customer driver has a great importance to improve sales and create a good image of the company. As far as we know, none of these aspects have been treated properly in the VRP literature.

\section{The multi-period distribution problem}

\subsection{Definition}

The idea is to create a multi-objective VRP that models in a closer way the real distribution problems, since the basic model is rarely applied in real problems due to its simplicity.

The basic model has an objective function that is centered in cost minimization. Distribution costs are, for many industries a significant portion of their total costs, but, they are not the only source of concern. In real business environments, companies face a complex variety of problems and the solutions for these problems are, in many cases, contradictory. There are conflicts of interest that must be managed and the way the conflicts are solved may be crucial for the success of the distribution policies.

Here we will try to reflect these concerns of a firm when designing its distribution strategies. We assume that the firm is responsible for the distribution of its own products. Therefore, there are no questions of outsourcing to be handled. Another assumption is that these firms face the pressures of a competitive market making them concerned both on consumer satisfaction and internal efficiency.

As far as we know, the only multiple objective VRP model considered in the literature is the one by Lee and Ueng (99). These authors developed an integer linear model that searches for the shortest travel path and balances driver's load simultaneously. The work is measured in terms of traveling and loading time. The objective function is the weighted sum of the two objectives. This second objective minimizes the difference between the working time of each vehicle and the working time of the vehicle with the shortest working time. Our paper 
introduces a different measure of work and of balance, multiple periods and a third objective.

\subsection{The multi-objective model}

In this section the motivation for the three objectives will be explained and also the assumptions of the model.

The classical VRP considers only one period and chooses the optimal routes for that period. Here we will introduce more periods by considering a week length of analysis. Each day we have a different set of clients to serve and quantities to deliver. The reason for this assumption is that in many industries we see that customers have low demands in terms of quantities but demand very frequently, tending to maintain low levels of stock. The just-in-time distribution is a tendency in many businesses to lower stock handling costs. This is particularly trueon the food and beverage industry. Since they may distribute their products to bars, small stores or larger supermarkets, these companies face very different types of customers. Some place orders almost every day and others place fewer orders but order higher quantities each time. Some days might also have higher demand like Fridays or Mondays.

As we will show later on, considering a multi-period model is essential to approach reality. In our model one of the objectives will be to minimize worklevels along several periods and not on a one period basis.

Other assumptions of the model are:

- All the demand is satisfied in the same day that it is required and not on any other day of the week.

- Only unload is done at each client.

- The number of vehicles is fixed and there are no fixed costs associated with the use of the vehicles. They all have the same capacity. Moreover, the number of vehicles available is enough to satisfy all the demand.

- Another assumption is that the cost matrix is known and fixed, independently of the day or the quantity loaded. The location of the clients is known.

- Each vehicle is assigned to a driver. For now we consider that they work every day in the period in question.

- One vehicle can only be used once a day and the time it takes to deliver the full capacity is less than a working day.

- Finally, we assume that each vehicle is driven always by the same driver. If we minimize differences in vehicles load we will balance employers work.

The way we measure work is not in terms of distance traveled but in quantities delivered. This way of dealing with work is based on the assumption that the work effort is centered on the unloading of the truck at the clients location, and not so much on the distance traveled. This measure could be changed into 
travel and unloading time. The choice of the type of measure will strongly depend on the specific industry we are facing. Our assumption makes sense for cases where products are distributed in a city versus others where distribution is through countries.

In the next sections the objectives and its formulations will be presented in detail. The following data is considered on the formulation:

$i, I \quad$ index and set of nodes, $I=1, \ldots, n$ where 1 is the depot and 2 to $n$ are the clients locations;

$k, K \quad$ index and set of vehicles, $K=1, \ldots, m$;

$t, T \quad$ index and set of days which represent the period, $T=1, \ldots, p$;

$T_{i} \quad$ set of days where client $i$ has a demand that is greater than zero, $i=2, \ldots, n$;

$q_{i}^{t} \quad$ demand of customer $i$ on day $t$ with $i=1, \ldots, n$ and $t=1, \ldots, p$;

$c_{i j} \quad$ the cost of going from $i$ to $j$, this is a fixed matrix with $i=1, \ldots, n$ and $j=1, \ldots, n$;

$Q \quad$ capacity of a vehicle.

The variables of the model are:

$x_{i j k}^{t}= \begin{cases}1, & \text { if vehicle } k \text { visits customer } j \text { immediatly after customer } i \text { on day } t \\ 0, & \text { otherwise }\end{cases}$

$y_{i k}^{t}= \begin{cases}1, & \text { if customer } i \text { is visited by vehicle } k \text { on day } t \\ 0, & \text { otherwise }\end{cases}$

\subsubsection{Objective A: Minimizing Cost}

Cost reduction is one of the biggest concerns in transportation and distribution management, but not the only one as we will see later. We want to find the route for each of the vehicles that will pass through the demand points in such a way as to satisfy all the demand with the smallest cost(or distance).

The formulation of this objective will be the same as the one used for the basic model but with a new parameter, $t$, representing the day of the week. 
Objective function :

$$
\operatorname{Min} \sum_{t=1}^{p} \sum_{i=1}^{n} \sum_{j=1}^{n} c_{i j} \sum_{k=1}^{m} x_{i j k}^{t}
$$

Subject to :

$$
\begin{aligned}
& \sum_{k=1}^{m} y_{i k}^{t}=1, \quad \forall i=2, \ldots, n ; \quad t \in T_{i} \\
& \sum_{k=1}^{m} y_{i k}^{t}=m, \quad \forall t \in T ; \quad i=1 \\
& \sum_{i=2}^{n} q_{i}^{t} y_{i k}^{t} \leq Q, \quad \forall k=1, \ldots, m ; \quad t=1, \ldots, p \\
& \sum_{j=1}^{n} x_{i j k}^{t}=\sum_{j=1}^{n} x_{j i k}^{t}=y_{i k}^{t} \\
& \forall i=2, \ldots, n ; k=1, \ldots, m ; t=1, \ldots, p \\
& \sum_{j, i \in S} x_{i j k}^{t} \leq|S|-1, \forall S \text { nonempty subset of }\{2, \ldots, n\} \\
& k=1, \ldots, m ; t=1, \ldots, p \\
& x_{i j k}^{t} \in\{0,1\} ; y_{i k}^{t} \in\{0,1\} \\
& \forall i=1, \ldots, n ; k=1, \ldots, m ; t=1, \ldots, p
\end{aligned}
$$

Constraints (1) to (6) are similar to the ones in the basic model.

Constraint (1) ensures that in the days where the clients have a positive demand, that client is visited by only one vehicle.

The second constraint forces that each day all vehicles go to the depot. Constraint (3) ensures that, the daily loading of a vehicle does not exceed its capacity.

Constraint (4) guarantees that if the vehicle enters a node, on day $t$, it also has to leave that node, on the same day.

Finally constraint (5) avoids subtours, but now not only for each vehicle but also for each day. The subtour elimination constraint represents an exponential number of constraints.

The problem with this constraint is that its number grows exponentially with $n$. Therefore, as it is usually done in the basic model, a weak form of the subtour elimination constraint is introduced to be able to solve it by a commercial software.

Desrochers \& Laporte (91) presented a weak form of the subtour elimination constraint for the TSP, this relaxation does not guarantee the elimination of subtours for large problems. Here, we will transform this weak form so that it can be applied to the VRP. The weak form proposed by Desrochers \& Laporte (91) for the TSP, is the following:

Consider the variable $u_{j}$ which represents the position of node $j$ in the tour, assume that every tour starts at 1 . Let $x_{i j}$ be the binomial variable that equals 
1 if the salesman goes from $i$ to $j$ and 0 otherwise. Then the constraint is:

$$
u_{j} \geq u_{i}+x_{i j}-(n-2)\left(1-x_{i j}\right)+(n-3) x_{j i} \quad \forall i, j: j \neq i \text { and } j>1
$$

and these other two, which are specific for the TSP:

$$
\begin{aligned}
& u_{j} \leq n-1-(n-2) x_{1 j} \quad \forall j, j>1 \\
& u_{j} \geq 1+(n-2) x_{j 1} \quad \forall j, j>1
\end{aligned}
$$

Transforming the previous for our VRP model we have:

Let $u_{j k}^{t}$ be the position of node $j$ in the tour made by vehicle $k$ on day $t$. The new constraint is:

$$
\begin{gathered}
u_{j k}^{t} \quad \geq \quad u_{i k}^{t}+x_{i j k}^{t}-(n-2)\left(1-x_{i j k}^{t}\right)+(n-3) x_{j i k}^{t} \\
\forall j \neq i \text { and } j>1, t \in T \text { and } k \in K
\end{gathered}
$$

This restriction holds even if $i$ and $j$ are not in the same route. If $i$ and $j$ are not on the same route on day $t$ then, the variable $x$ will equal $0\left(x_{i j k}^{t}=0\right.$ and $\left.x_{j i k}^{t}=0\right)$ and the constraint becomes:

$u_{j k}^{t} \geq u_{i k}^{t}+0-(n-2)(1-0)+(n-3) \times 0$

$u_{j k}^{t} \geq u_{i k}^{t}+2-n$

This is always true, the position of a node is always higher or equal than 1 . In the worst case all the $n$ nodes are in this tour and node $i$ is the last to be visited, $u_{i k}^{t}=n-1$ and $u_{j k}^{t} \geq 1$.

With this weak formulation we reduce the number of constraints and enable a commercial software to solve the problem.

The last constraints (6) define all variables as binary.

\subsubsection{Objective B: Balance Worklevels}

The second objective function, seeks to balance the work between vehicles. The idea of making a multi-objective model that balances the work and minimizes cost has already been explored in the work of Lee and Ueng (99). In that paper the way the work is balanced is by minimizing the difference between the work of each vehicle and the work of the vehicle with the lowest work level, where the work was measured in terms of traveling and loading time.

In our model we considered the measure of the work related with the volume transported during all periods. Ths is particularly important in industries, like food and beverages, where is everyday more common to have a percentage of the remuneration related with the amount of sales and distributed products. Therefore, to measure the equilibrium of the routes we will consider a statistic, the standard deviation, since it is a well known measure of deviations or spread around the mean. 
The standard deviation of the work of each vehicle at the end of the period is minimized. The model allows a vehicle to work more than another on a given day as long as the total work of a vehicle at the end of the period is balanced. The problem of using the standard deviation as an objective function is that we no longer have a linear function and this adds complexity to the model.

The total work of each vehicle will be represented by $w_{k}$, where:

$$
w_{k}=\sum_{t=1}^{p} \sum_{i=1}^{n} q_{i}^{t} y_{i k}^{t} \quad \forall k=1, \ldots, m
$$

and, the objective function will be:

$$
\operatorname{Min}\{\operatorname{StdDev}\}=\operatorname{Min}\left\{\sqrt{\frac{\sum_{k=1}^{m} w_{k}^{2}}{m}-\left(\frac{\sum_{k=1}^{m} w_{k}}{m}\right)^{2}}\right\}
$$

Constraints (1) to (6) in this objective are the same as in the previous model and we add a new constraint:

$$
\sum_{k=1}^{m} y_{i k}^{t}=0, \quad \forall \quad i=2, \ldots, n \text { and } t \notin T_{i}
$$

Constraint (7) prohibits a vehicle to visit a client on a day where he has zero demand. In the first objective we did not need this constraint since the objective was to minimize distance and the solution did not go through empty nodes. Now, distance is not included in the objective and going into a node with no demand is not penalizing the objective function $\mathrm{B}$.

\subsubsection{Objective C: Marketing Objective}

In this model, each driver will try to serve always the same customer. This marketing policy is giving emphasis to the personal relationship between drivers and customers as a way to improve customer service. Since we have other constraints such as capacity and number of vehicles, we want this to happen the maximum number of times possible. This requirement may have to be sacrificed but we will try to enforce this at least to the best clients. Therefore, the idea is: the better the client is the more interest we have in maintaining the same driver. This policy is becoming relevant in many industries, in particular the food and beverages, since the driver also performs commercial tasks.

In terms of mathematical formulation, the third objective works as follows: For each client we have a set of pairs of days with positive demand, $T_{i}$, for each pair of days $(g, h)$ in $T_{i}$ (with $g \neq h$ ) we want to minimize the difference in the assignment to a vehicle $k$. The objective is to minimize $\left|y_{i k}^{g}-y_{i k}^{h}\right|$.

The objective function will be: 


$$
\operatorname{Min} \sum_{i=1}^{n} \sum_{k=1}^{m} \sum_{(g, h) \in T_{i}}\left|y_{i k}^{g}-y_{i k}^{h}\right|
$$

The importance is given by the total demand in the period, therefore a weight is introduced by the total amount ordered by each client. The objective function becomes:

$$
\operatorname{Min}\left\{\sum_{i=1}^{n} \sum_{\substack { k=1 \\
\begin{subarray}{c}{g, h \in T_{i} \\
g<h{ k = 1 \\
\begin{subarray} { c } { g , h \in T _ { i } \\
g < h } }\end{subarray}}^{m}\left[\left(\sum_{t=1}^{p} q_{i}\right) \times\left|y_{i k}^{g}-y_{i k}^{h}\right|\right]\right\}
$$

The importance of a customer is measured in terms of sales. In some cases other measures could be used to classify the goodness of a client. For example, frequency of orders, credit history.

This objective function is again non linear. Constraints are the same as in section 3.2.2.

\subsubsection{Summary of the complete model.}

As mentioned before, when designing the distribution routes the three objectives can be important, an appropriate model is a multi-objective one, which can be described as: 
$\underbrace{\operatorname{Min}(\text { total cost })}_{\mathrm{A}} ; \underbrace{\operatorname{Min}(\neq \text { in work levels })}_{\mathrm{B}}$ and $\underbrace{\operatorname{Min}(\neq \text { in client service })}_{\mathrm{C}}$

Objective function :

Subject to :

$$
\begin{aligned}
& \text { A } \operatorname{Min} \sum_{t=1}^{p} \sum_{i=1}^{n} \sum_{j=1}^{n} c_{i j} \sum_{k=1}^{m} x_{i j k}^{t} ; \\
& \text { В } \operatorname{Min}\left\{\sqrt{\frac{\sum_{k=1}^{m} w_{k}^{2}}{m}-\left(\frac{\sum_{k=1}^{m} w_{k}}{m}\right)^{2}}\right\} ; \\
& \text { C } \operatorname{Min}\left\{\sum_{i=1}^{n} \sum_{\substack { k=1 \\
\begin{subarray}{c}{g, h \in T_{i} \\
g<h{ k = 1 \\
\begin{subarray} { c } { g , h \in T _ { i } \\
g < h } }\end{subarray}}^{m}\left[\left(\sum_{t=1}^{p} q_{i}\right) \times\left|y_{i k}^{g}-y_{i k}^{h}\right|\right]\right\}
\end{aligned}
$$

$$
\begin{aligned}
& \sum_{k=1}^{m} y_{i k}^{t}=1, \quad \forall i=2, \ldots, n ; \quad t \in T_{i} \\
& \sum_{k=1}^{m} y_{1 k}^{t}=m, \quad \forall t \in T \\
& \sum_{i=1}^{n} q_{i}^{t} y_{i k}^{t} \leq Q, \quad \forall k=1, \ldots, m ; t=1, \ldots, p \\
& \sum_{j=1}^{n} x_{i j k}^{t}=\sum_{j=1}^{n} x_{j i k}^{t}=y_{i k}^{t}, \\
& \forall i=2, \ldots, n ; k=1, \ldots, m ; t=1, \ldots, p \\
& \sum_{j, i \in S} x_{i j k}^{t} \leq|S|-1, \forall S \text { nonempty subset of }\{2, \ldots, n\} \\
& k=1, \ldots, m ; t=1, \ldots, p \\
& x_{i j k}^{t} \in\{0,1\} ; \quad y_{i k}^{t} \in\{0,1\} \\
& \forall i=1, \ldots, n ; k=1, \ldots, m ; t=1, \ldots, p \\
& \sum_{k=1}^{m} y_{i k}^{t}=0, \quad \forall i=2, \ldots, n ; t \notin T_{i} \\
& \text { and } \sum_{t=1}^{p} \sum_{i=1}^{n} q_{i}^{t} y_{i k}^{t}=w_{k} \quad \forall k=1, \ldots, m
\end{aligned}
$$

To guarantee that the assumption on the number of the vehicles holds we can calculate the number of vehicles required.

$$
m=\left\lceil\frac{\operatorname{Max}\left(\sum_{i=1}^{n} q_{i}^{1}, \ldots, \sum_{i=1}^{n} q_{i}^{p}\right)}{Q}\right\rceil
$$

Ideally, we would like to find the solution that would be optimal for the three objectives at the same time. In multi-objective programming this solution point 
rarely exists. So, we would like to find solutions that are closer to this ideal point.

In the multi-objective optimization an important relation is the dominance relation.

Let $\left(z_{1}\right)$ and $\left(z_{2}\right)$ be two solutions of a multi-objective problem with $R$ objectives. We say that:

Solution $\left(z_{1}\right)$ dominates $\left(z_{2}\right) \quad$ if $\quad z_{1 r} \leq z_{2 r} \quad \forall$ objectives $r$ in $\{1, \ldots, R\}$; $z_{1 r}<z_{2 r}$ for at least one $r$ and $\left(z_{1}\right) \neq\left(z_{2}\right)$. A feasible solution is efficient if it is non-dominated.

Based on this concept we will optimize the three objective functions to find non-dominated solutions. The choice among these non-dominated solutions is determined by the decision maker's preferences among the multiple objective. In the next section these preferences are introduced as weights.

\subsection{Weighting function approach}

An usual approach to solve multi-objective problems is to consider all the objectives in the same objective function. The simplest method to do this is the Objective Weighting, where a weight is given to each of the objectives and the sum of them is optimized. The objective function $Z$ is a weighted sum of the single objectives $f_{\lambda}, \lambda=1, \ldots, e$ and $e$ is the number of objectives.

$Z=\sum_{\lambda=1}^{e} \alpha_{\lambda} f_{\lambda}(x)$ where $\quad x \in X$ and $X$ represents the feasible region and $\sum_{\lambda=1}^{e} \alpha_{\lambda}=1$.

In this method the optimal solution is controlled by the weight vector $\alpha$. The preference of an objective can be changed by modifying the corresponding weight.

The use of this method may lead to Pareto-Optimal solutions but has some drawbacks. The solution is very sensitive to the weights that have been defined. The problem lies also on having objectives with different variables and scales. In our case, for example we are adding costs with standard deviations and quantities, forcing to use cost measures for the second and third objectives. This, in practical terms increases the complexity of the decision process.

To apply this method to our model we have to define objective $\mathrm{B}$ and $\mathrm{C}$ in terms of costs and in a similar scale.

Let $c_{B}$ be the cost per unit of standard deviation, this can be interpreted as the cost for a firm of having disequilibrium in routes. And $c_{C}$ unit cost to the company for not serving a client with the same salesman. This could also be interpreted as the penalty for not going to the same client.

The weighted objective function with weights $\alpha_{1}, \alpha_{2}, \alpha_{3} \geq 0$, such that 
$\sum_{\lambda=1}^{3} \alpha_{\lambda}=1$ is as follows:

$$
\begin{aligned}
& \operatorname{Min} \alpha_{1} \sum_{t=1}^{p} \sum_{i=1}^{n} \sum_{j=1}^{n} c_{i j} \sum_{k=1}^{m} x_{i j k}^{t}+\alpha_{2} c_{B}\left\{\sqrt{\frac{\sum_{k=1}^{m} w_{k}^{2}}{m}-\left(\frac{\sum_{k=1}^{m} w_{k}}{m}\right)^{2}}\right\}+ \\
& +\alpha_{3} c_{C}\left\{\sum_{i=1}^{n} \sum_{\substack{k=1 \\
m}}^{m}\left[\left(\sum_{\substack{g, h \in T_{i} \\
g \in h}}^{p} q_{i}\right) \times\left|y_{i k}^{g}-y_{i k}^{h}\right|\right]\right\}
\end{aligned}
$$

Subject to :

$$
\begin{aligned}
& \text { (1) to }(7) \\
& \text { and } \sum_{t=1}^{p} \sum_{i=1}^{n} q_{i}^{t} y_{i k}^{t}=w_{k} \quad \forall k=1, \ldots, m
\end{aligned}
$$

To use this method we face two problems: The first one is related with the weight that will be given to each objective and the second problem is the comparability between objectives through the transformation of the objectives into identical scales.

\section{Solution Approach}

In this section we will present solutions for the above problem using a small example.

The Lingo 2.1 Industrial version was used to run the algorithms. Lingo is a mathematical modeling language that solves linear and nonlinear problems. For the linear problems LINGO uses Primal and Dual Simplex solvers or a Barrier solver. The Barrier solver utilizes a barrier or interior point method to solve linear models. The non-linear solver is based on the Generalized Reduced Gradient algorithm. The method does not guarantee that the solution found is a global optimum.

The example considers 5 clients, 2 vehicles and 3 days. The option to use an example with only three days is just to have a short computational time. And, on the other hand the number of days is large enough to allow the analysis of the results. The demand and distances matrices used for the example can be see in Appendix 1.

Figures 1 to 3 represent the solutions for 3 scenarios, corresponding to the optimization of the 3 objective functions (A, B and C) independently. In these pictures one can see the optimal routes and the total work of each vehicle. As can be observed all the routes are different depending on the objective we want to minimize, and, in general, the three objectives are contradictory.

For example, when we minimize objective A, we obtain unbalanced routes. The total work of vehicle 1 is 230 and vehicle 2, 90. Or, when we balance the work we have that client 4 is served by different vehicles on the second and third days. Table 1 shows the results obtained for the three objective functions in the 3 cases. All the solutions are non-dominant. That is, in all the solutions 


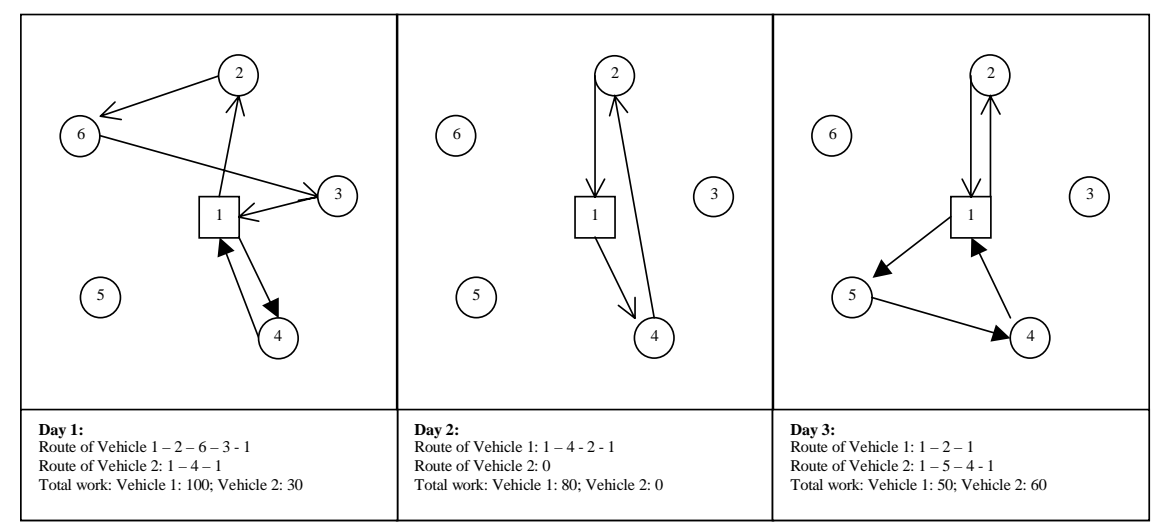

Figure 1: Optimal Solution: Objective A

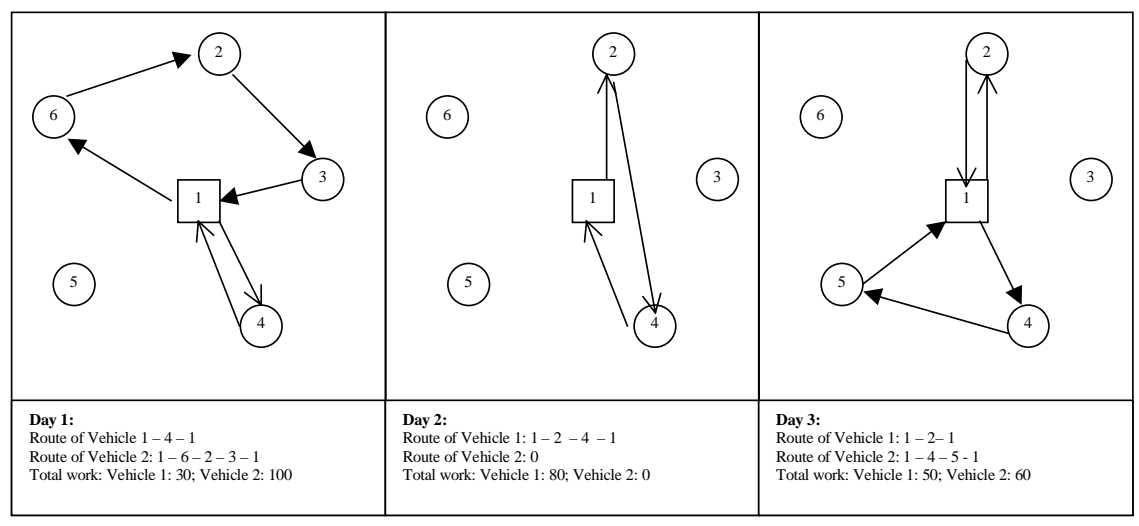

Figure 2: Optimal Solution: Objective B

there is at least one objective that has a better value on another solution.

\begin{tabular}{|c|c|c|c|}
\hline & $A$ & $B$ & $C$ \\
\hline Optimize A & $\mathbf{1 3 2 9 7}$ & 70 & 280 \\
\hline Optimize B & 14428 & $\mathbf{0}$ & 880 \\
\hline Optimize C & 15381 & 50 & $\mathbf{0}$ \\
\hline \multicolumn{4}{|c}{ Table 1}
\end{tabular}

In Figure 4, we can see the same results has in the Table 1. None of these triangles is totally covered by the other. Which means that none of the solutions is dominated by another.

The next step will be to implement the weighting method approach to the same example, we will call it objective M. For this implementation we need to define the weights and the costs associated with objective $\mathrm{B}$ and $\mathrm{C}$.

Let $c_{B}=200$ and $c_{C}=50$

Consider 2 examples: M1 and M2. The only difference between these examples is the weight given to each objective. In M1 all the objectives are equally important, all $\alpha$ are equal to $1 / 3$ and in M2 the weights are $\alpha_{1}=0,5 ; \alpha_{2}=$ 


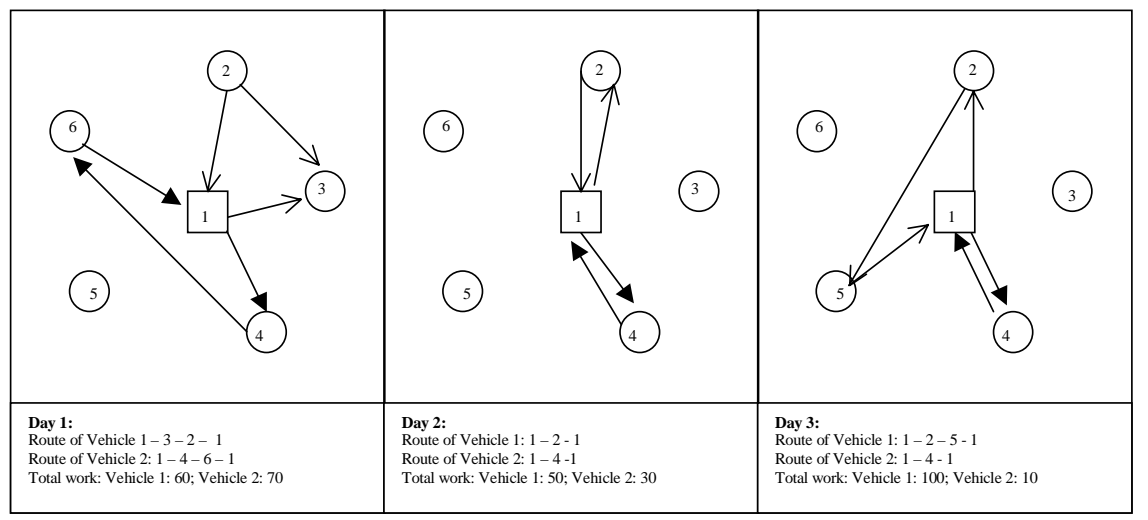

Figure 3: Optimal Solution: Objective C

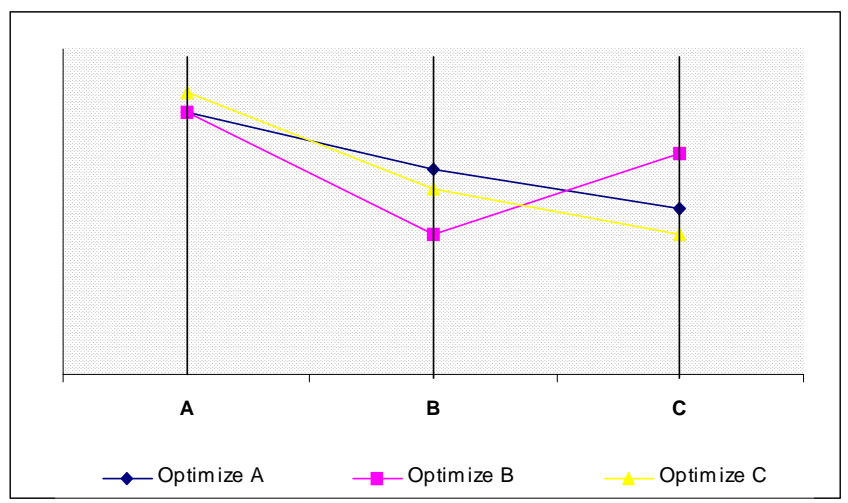

Figure 4: Graphical representation of the solutions 


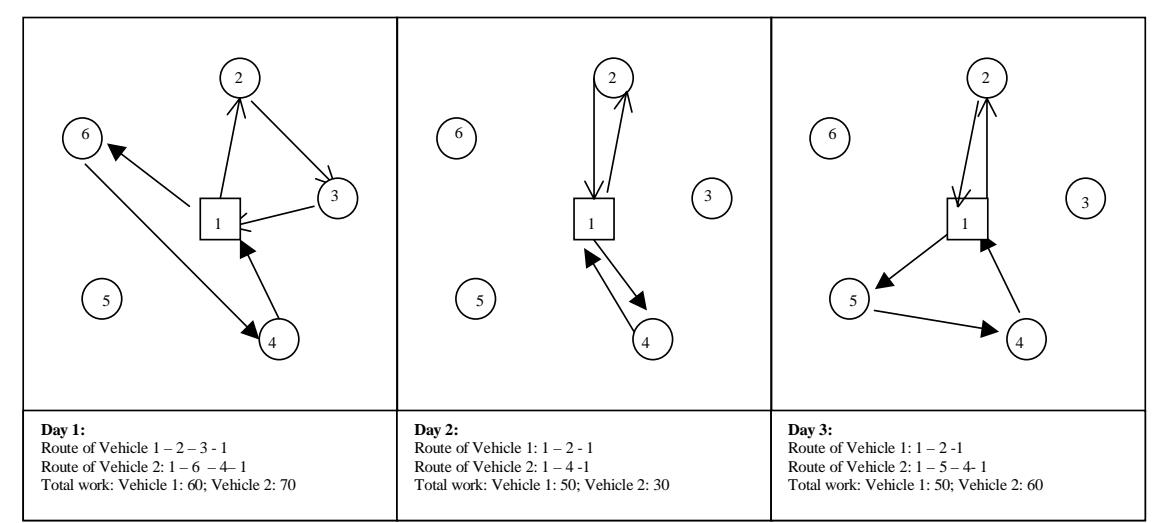

Figure 5: Optimal Solution: Objective M1

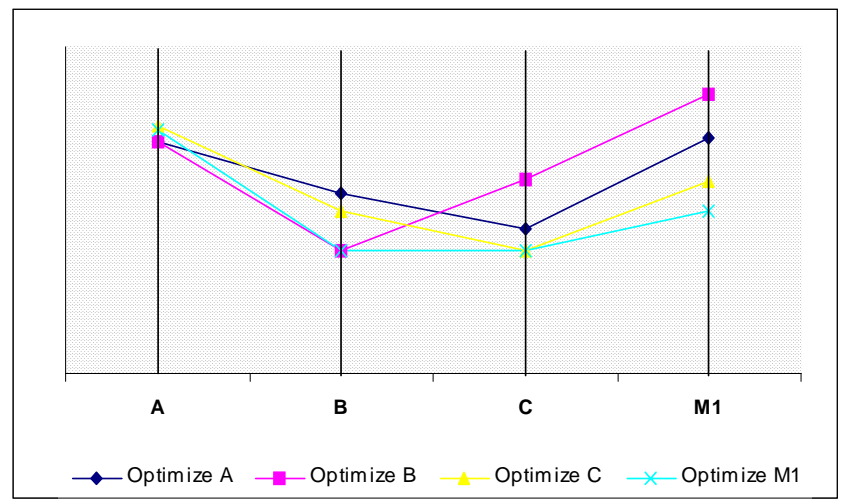

Figure 6: Graphical representations of the solutions

$\alpha_{3}=0,25$. The solution of problem M1 (Figure 5) is different from the one found when considering the objectives separately. The values of the different objectives can be seen in Table 2 and Figure 6.

\begin{tabular}{|c|c|c|c|c|c|}
\hline & A & B & C & M1 & M2 \\
\hline OptimizeA & $\mathbf{1 3 2 9 7}$ & 14000 & 14000 & 13752 & 13649 \\
\hline Optimize B & 13317 & 0 & 44000 & 19087 & 18099 \\
\hline Optimize C & 15381 & 10000 & 0 & 8452 & 10091 \\
\hline Optimize M1 & 14721 & 0 & 0 & 4902 & 7361 \\
\hline Optimize M2 & 14701 & 2000 & 0 & 5561 & 7831 \\
\hline
\end{tabular}

The solutions obtained when optimizing objective M1 and M2 dominate the one that results from optimizing objective $\mathrm{C}$. It is an optimal solution for the objective $\mathrm{C}$ and produces better values for objectives $\mathrm{A}$ and B. For the nonlinear objective functions we cannot guarantee that the solution is a global optimum. The solution found for objective M2 is a local optimum, the value of the objective function M2 is higher than the one we would obtain applying the solution found for M1. 
If we try to increase the size of the example, by adding one more day, the computational complexity increases exponentially. To show an example of the complexity of this problem, when trying to solve an example with 4 days, 2 vehicles, and 5 clients, on a PC (Pentium , 32 MB RAM ), after 2 days the LINGO had not been able to find a solution. These computational times are not compatible with a firm's decision processes.

\section{Summary and Conclusions}

This work presents a new extension of the VRP, which tries to reflect more the "real world" situations and business concerns. The model is a multi-objective combinatorial optimization problem with three objectives: minimizing cost, balancing work and improving customer service. We have implemented the model on a small example and found some non-dominated solutions.

The results obtained matched our expectations: The three objectives are contradictory and, when optimizing one of them we obtain bad solutions for the other two. When using the weighted method as an approach to solve the multi-objective issue we face two problems: the first is that the costs associated with $\mathrm{B}$ and $\mathrm{C}$ are artificial and influence the results; and second, the definition of preferences for each objective also influence results.Just by analysing such a small example, we were able to conclude the importance of the inclusion of multiobjectives in the multiperiod model.

Since the idea is to approximate the model to reality we need to overcome the complexity of the model and the time to find solutions to be able to apply it to real cases and help the user to take decisions. Therefore, as further work we will develop an heuristic approach, based on a technique known as iterated local search, that we hope that it will enable us to obtain good results in shorter running times.

For further research we also thought about extending this model to include stochastic demand in order to compete with the new trend of on-line delivery systems arising on the area of e-commerce.

Acknowledgement : We would also like to thank Daniel Serra and Frederic Udina for their valuable suggestions during the elaboration of this article. This research was partially funded by the Ministério da Ciência e da Tecnologia, FCT Programa Praxis XXI, Portugal (for the first author) and Ministerio de Ciencia y Tecnología, Spain (BEC 2000-1027) (for the second author).

\section{References}

[1] Aarts, E. and Lenstra, J.K. (1997), "Local search Combinatorial Optimization", Wiley \& Sons Ltd.

[2] Atkinson,J.B. (1998), "A greedy randomized search heuristic for timeconstrained vehicle scheduling and the incorporation of a learning strategy", Journal of the Operational Research Society 49, 700-708. 
[3] Bard, J.F., Huang, L., Dror, M. and Jaillet, P. (1998), "A branch and cut algorithm for the VRP with satellite facilities", IIE Transactions 30, 821-834.

[4] Brandão, J., Mercer, A. (1997), "A tabu search for the multi-trip vehicle routing and scheduling problem", European Journal of Operational Research 100, 180-191.

[5] Clarke, G. and Wright, J. (1964), "Scheduling of vehicles from a central depot to a number of delivery points", Operations Research 12, 568-581.

[6] Crainic, T.G., Laporte, G(1998)., "Fleet Management and Logistics", Centre for Research on Transportation, Kluwer Academic Publishers.

[7] Dantzing, G.B. and Ramser, J.H. (1959), "The truck dispatching problem", Management Science 6, 80-91.

[8] Dell'Amico, M., Fischetti, M. and Toth, P. (1993), "Heuristic Algorithms for the Multiple Depot Vehicle Scheduling Problem", Management Science Vol.39, No.1, 115-125.

[9] Desrochers, Laporte, G. (1991), Operational Research Letters, February.

[10] Desrochers, M., Desroisers, J. and Solomon, M.M. (1992), "A new Optimization algorithm for the vehicle routing problem with time windows", Operations Research 40, 342-354.

[11] Desrosiers, J. ,Dumas, Y., Solomon, M.M., and Soumis, F. (1996), "The routing and Scheduling" in Networks and Distribution, Handbooks in OR and MS, North-Holland, Amsterdam.

[12] Fisher, M.L., Jaikumar (1978), "A decomposition algorithm for large-scale vehicle routing", report 78-11-05, Department of decision Sciences, The Wharton School, University of Pennsylvania, Philadelphia [12;2,3].

[13] Fisher, M.L., Jaikumar (1981), "A generalized assignment heuristic for vehicle routing", Networks 11, 109-124[12:2,4].

[14] Fisher, M.L. (1996), "Vehicle Routing", in Networks and Distribution, Handbooks in OR and MS, North-Holland, Amsterdam.

[15] Gendreau, M., Laporte, G., Séguin, R. (1996), "Stochastic vehicle routing", European Journal of Operational Research 88, 3-12.

[16] Golden, B.L., Wasil, E.A. (1987), "Computerized vehicle routing in the soft drink industry", Operations Research 36, No.1, 6-17.

[17] Gouveia, L. (1995), "A result on projection for the Vehicle Routing Problem", European Journal of Operational Research 85, 610-624.

[18] Kohl, N., Desrosiers, J., Madsen, O.B.G., Solomon, M. and Soumis, F. (1999), "2-Path Cuts for the Vehicle Routing Problem with Time Windows", Transportation Science, Vol.33, No.1, 101-116. 
[19] Kulkarni, R.V. and Bhave, P.R. (1985), "Integer programming formulations of vehicle routing problems", European Journal of Operational Research 20, 58-67.

[20] Laporte, G. and Osman, I.H. (1995), "Routing problems: A bibliography", Annals of Operations Research.

[21] Laporte, G. (1992), " The vehicle routing problem: An overview of exact and approximate algorithms", European Journal of Operational Research $59,345-358$.

[22] Lawer, E.L., Lenstra, J.K., Rinnooy Kan, A.H.G. and Shmoys, D.B. (1985), "The Traveling Salesman Problem", Wiley \& Sons Ltd.

[23] Lee, T. and Ueng, J. (1999), "A study of vehicle routing problems with load-balancing", International Journal of Physical Distribution 83 Logistics Management, Vol.29, No.10, 646-658.

[24] Mingozzi, A., Giorgi, S. and Baldacci, R. (1999), "An Exact Method for the VRP with Backhauls", Transportation Science, Vol.33, No.3, 315-329.

[25] Ochi, L.S., Vianna, D.S., Drummond, L.M.A. and Victor, O.A. (1998), "Evolutionary Hybrid Metaheuristic for Solving the VRP with heterogeneous fleet", UFF - Brazil.

[26] Partyka, J.G., Hall, R.W.(2000), "On the Road to Service", OR/MS Today, August 2000, 26-35.

[27] Ringuest, J.L., "Multi-Objective Optimization: Computational Considerations", Kluwer Academic Publishers (1992).

[28] Sariklis, D., Powell, S. (2000), "A heuristic method for the open vehicle routing problem", Journal of the Operational Research Society 51, 564-573.

[29] Taillard, E.D., Laporte, G. and Gendreau, M. (1996), "VR with Multiple Use of Vehicles", Journal of the Operational Research Society 65, 10651070 .

[30] Taillard, E.D., Badeau, P., Gendreau, M., Guertin, F. and Potvin, J. (1997), "A tabu search heuristic for the VRP with soft Time windows", Transportation Science, Vol.31, No.2, 170-186. 